\title{
REABILITAÇÃO DE INCISIVOS DECÍDUOS SUPERIORES COM NÚCLEO METÁLICO E COROA ESTÉTICA: RELATO DE CASO CLÍNICO
}

\author{
ESTHETIC REHABILITATION OF ANTERIOR PRIMARY TEETH \\ USING METAL POST AND ESTHETIC CROWN: A CASE REPORT
}

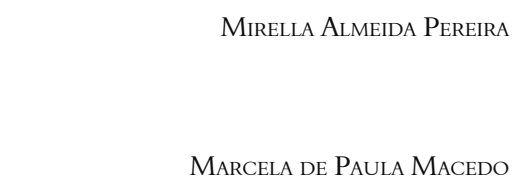

Claudia Valeria de Sousa Resende Penido

\section{Resumo}

Introdução: A cárie aguda da infância é a principal causa de destruição e perda de dentes anteriores. Vários materiais restauradores foram utilizados ao longo dos anos na odontologia, muitos já estão em desuso como coroas de aço pré-fabricadas e coroas de policarbonato. Porém, outros materiais têm sido desenvolvidos, os quais atualmente permitem reconstruções coronárias extensas com coroas de celulóide, coroas feitas a partir de um dente natural, as chamadas coroas biológicas, além das resinas diretas e indiretas. Objetivo: Este trabalho tem como objetivo apresentar um caso clínico onde, após tratamento endodôntico, foram confeccionados núcleos metálicos fundidos, e, em seguida, coroas acrílicas prensadas. Conclusão: esta técnica é uma alternativa de reabilitação de dentes decíduos extensamente destruídos, de fácil execução e excelentes resultados estéticos e funcionais.

Palavras-chave: REABILITAÇÃo BUCAL; DENTIÇÃO DECÍDUA; ESTÉTICA DENTÁRIA.
Pontifícia Universidade Católica de Minas Gerais Graduada em Odontologia pela Pontifícia Universidade Católica de Minas Gerais

Graduada em Odontologia pela Pontifícia Universidade Católica de Minas Gerais

Pontifícia Universidade Católica de Minas Gerais. Professora Adjunto III da Pontifícia Universidade Católica de Minas Gerais

\section{Abstract}

Introduction: The acute childhood caries is the main cause of destruction and loss of anterior teeth. Various restorative materials were used over the years in Dentistry and many of them are already in disuse, as prefabricated steel crowns and polycarbonate crowns. However, others materials have been developed, which currently allow extensive coronary reconstructions with celluloid crowns, with crowns made from a natural tooth (called biological crowns) and with direct and indirect resins. Objective: This paper aims to present a case where after root canal treatment, primary anterior teeth were restored with metal posts and pressed acrylic crowns. Conclusion: This technique is a rehabilitation alternative to extensively destroyed of primary teeth. It is also easy to perform and has excellent cosmetic and functional results.

Keywords: MOUTH REHABILITATION; PRIMARY DENTITION; DENTAL ESTHETIC. 


\section{INTRODUÇÃO}

São muito frequentes na prática diária as situações em que os dentes decíduos são destruídos em uma idade precoce, devido aos casos de extensas lesões de cárie. A reconstrução desses elementos dentários é de extrema importância, pois evita que haja alterações nas funções da mastigação e da fala, além de prevenir o desenvolvimento de hábitos parafuncionais. Além disto, minimiza o surgimento de problemas psicológicos, que podem interferir na personalidade e comportamento da criança. ${ }^{1}$

Incisivos decíduos destruídos são difíceis de serem restaurados em razão do tamanho reduzido das coroas e da amplitude da câmara pulpar. Assim, torna-se muitas vezes necessária a utilização de reforço intrarradicular antes de reconstruir a porção coronária. Vários tipos de reforços intrarradiculares são utilizados em dentes decíduos anteriores, como os pinos pré-fabricados em fibra de vidro, pinos confeccionados com fio-ortodôntico, núcleos metálicos fundidos, preenchidos com resina composta, pinos biológicos, dentre outros. ${ }^{2}$

A coroa de resina acrílica prensada é uma opção por apresentar resultado estético favorável. Possibilita adequação da forma antes da cimentação, além de o reduzir tempo clínico da criança na cadeira odontológica. ${ }^{3}$ Diante das opções restauradoras, a escolha do profissional deve ser influenciada pelo comportamento da criança, pelo custo do material e também pela sua habilidade.

No presente trabalho é apresentado o caso clínico de uma criança, com coroas dentárias totalmente destruídas, que receberam núcleos metálicos fundidos, sobre os quais foram colocadas coroas de resina acrílica prensada.

\section{Relato de Caso}

Paciente do gênero masculino, 5 anos de idade, procurou por tratamento odontológico no Depto. de Odontologia da PUC/Minas, devido à extensa destruição coronária nos dentes 52, 51, 61 e 62 (Figura 1).

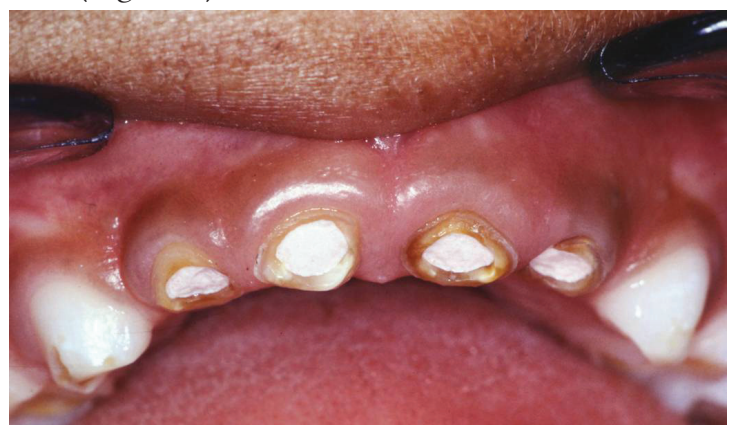

FIGURA 1: Extensa destruição coronária nos elementos 51,52,61,62
Ao exame clínico intrabucal observou-se dentição decídua completa, destruição coronária dos incisivos superiores e higiene bucal insatisfatória.

Os responsáveis foram informados a respeito do tratamento e da possibilidade de publicação dos dados e, então, assinaram o Termo de Consentimento Livre Esclarecido (TCLE).

Inicialmente foi realizado tratamento endodôntico dos quatro incisivos superiores, sendo obturados com pasta de óxido de zinco e eugenol puro (SS White ${ }^{\circledR}$ Artigos Dentários ${ }^{\circledR}$, Rio de janeiro, RJ, Brasil).

$\mathrm{Na}$ sequência, foi retirado $1 / 3$ do material obturador com brocas esféricas número 4 e 5 (KG Sorensen ${ }^{\circledR}$, Barueri, SP, Brasil) em baixa rotação, e realizado chanfrado no término cervical com broca diamantada número 2224 (KG Sorensen ${ }^{\circledR}$, Barueri, SP, Brasil), em alta rotação.

Foram preparados cones de madeira de forma que se adaptassem no interior dos canais. Os mesmos foram removidos e os procedimentos para moldagem foram realizados. Com o auxílio de seringa (Jon Produtos Odontológicos ${ }^{\circledR}$, São Paulo, SP, Brasil) o material à base de silicone leve foi levado, o Xantopren ${ }^{\circledR}$ (Heraeus Kulzer ${ }^{\circledR}$, São Paulo, SP, Brasil), ao interior dos canais. Em seguida, os cones de madeira previamente preparados foram inseridos de maneira que forçassem o material moldador a ocupar todo o espaço do canal preparado, sem o inconveniente da formação de bolhas (Fig. 2 A e B). Antes que o silicone leve catalisasse, moldou-se a região anterior da arcada superior com silicone pesado Optosil (Heraeus Kulzer®, São Paulo, SP, Brasil) utilizando moldeira parcial (Tecnodent ${ }^{\circledR}$, São Paulo, SP, Brasil). A moldagem obtida foi enviada ao laboratório, e os núcleos foram confeccionados com liga de prata DFL (La Croix ${ }^{\circledR}$, Rio de Janeiro, RJ, Brasil).

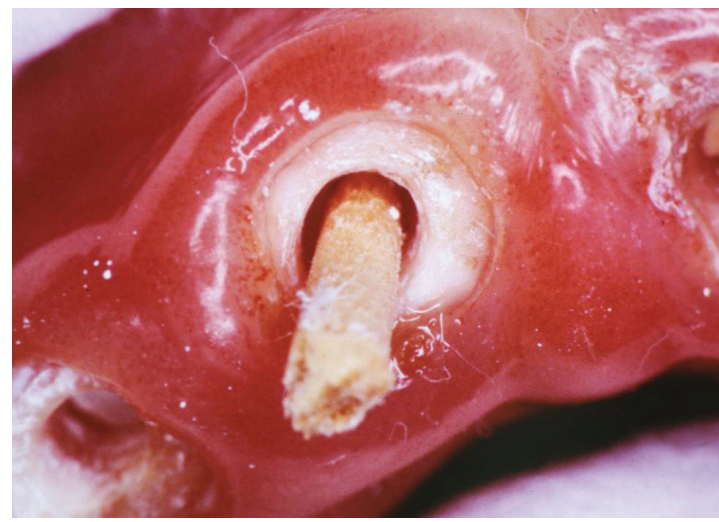

FIgURA 2: (A) Cone de madeira inserido no canal preparado. 


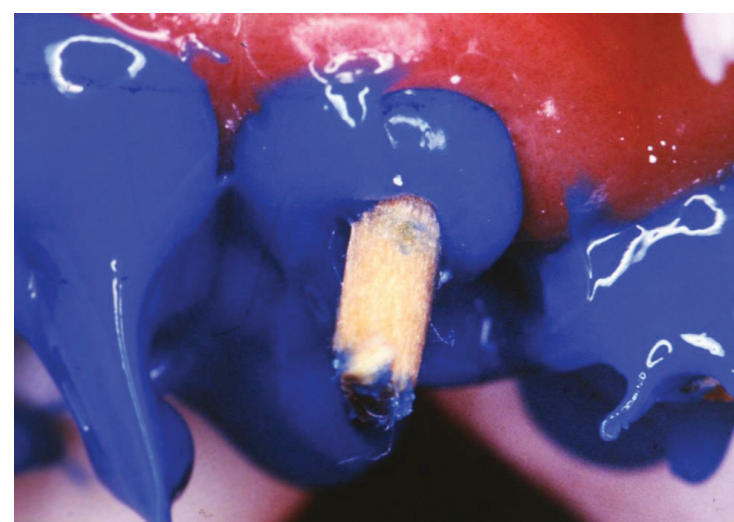

Figura 2:(B) Material de moldagem à base de silicone leve.

Os núcleos perfeitamente adaptados foram cimentados com cimento de ionômero de vidro tipo III Ketac-Cem (3M Espe ${ }^{\circledR}$, Seefeld, Baviera, Alemanha) (Figura 3 A e B).
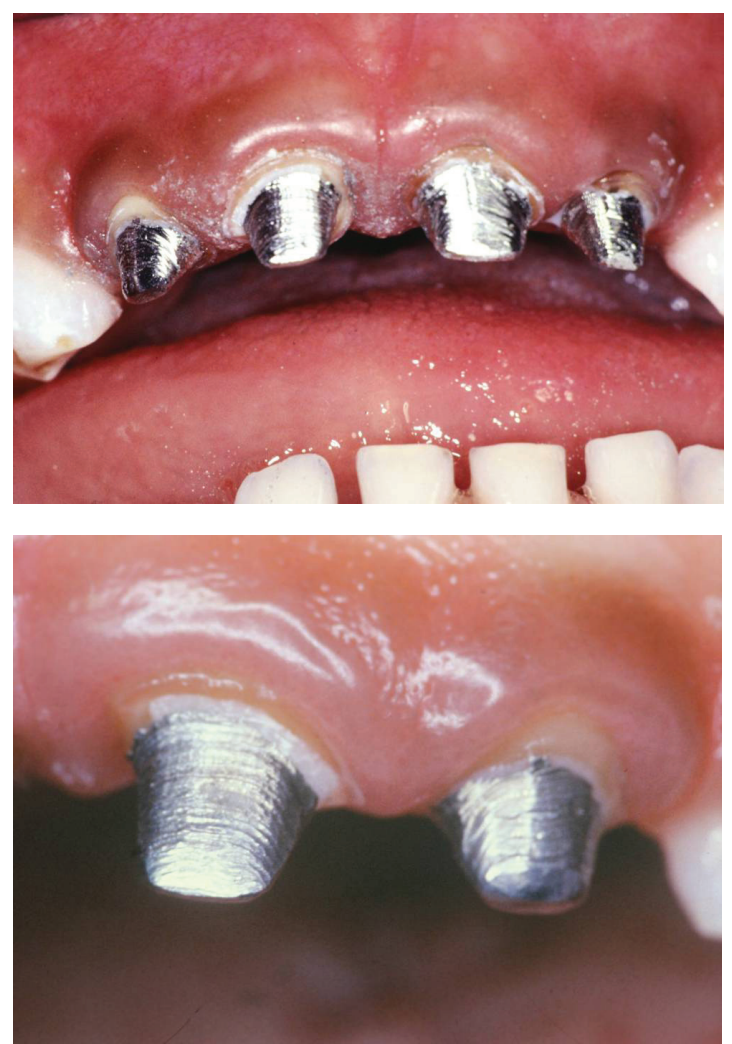

Figura 3 (A e B): Núcleos perfeitamente adaptados e cimentados.

Procedeu-se então a nova moldagem com Optosil e Xantopren para obtenção de novo modelo de trabalho onde foram confeccionadas coroas de acrílico prensadas. Figura 4 A e B). Para abreviar o tempo de atendimento clínico, já que o paciente era extremamente inquieto, este foi dispensado, tendo somente os núcleos em posição, sendo a responsável comunicada do retorno na mesma semana para instalação das coroas definitivas.

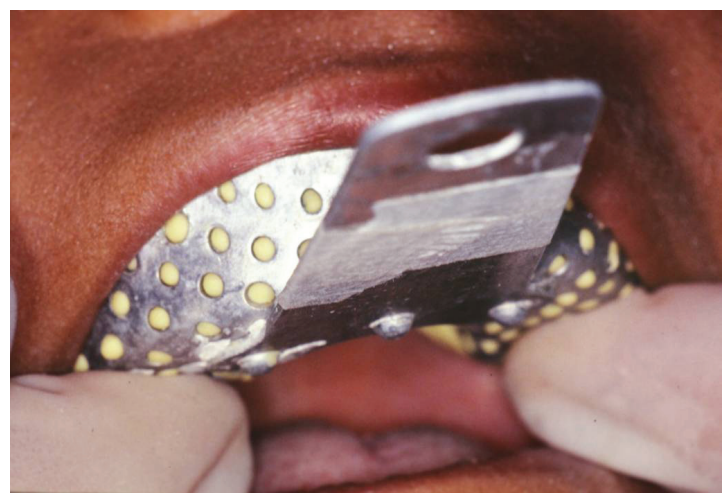

Figura 4: (A) Moldagem com silicone pesado.

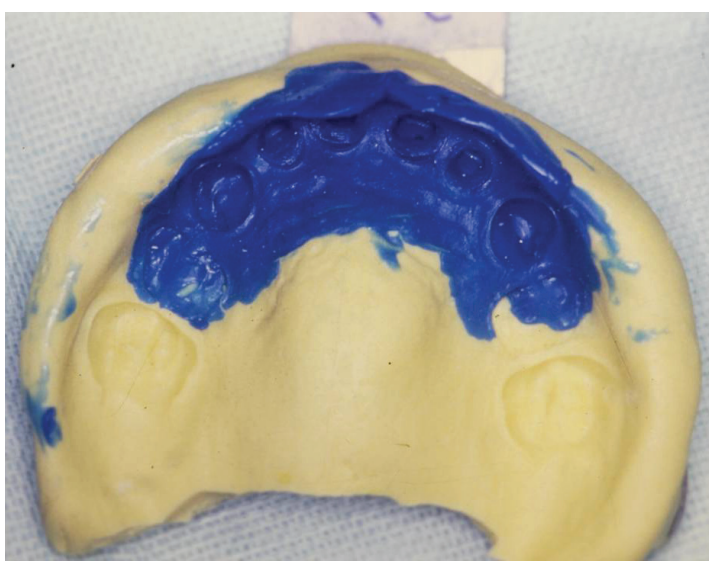

Figura 4: (B) Vista oclusal do molde obtido.

$\mathrm{Na}$ sessão subsequente, as coroas ajustadas na cavidade bucal foram cimentadas também com o cimento de ionômero de vidro tipo III Ketac-Cem (3M Espe ${ }^{\circledR}$, Seefeld, Baviera, Alemanha) (Figura 5 A e B).

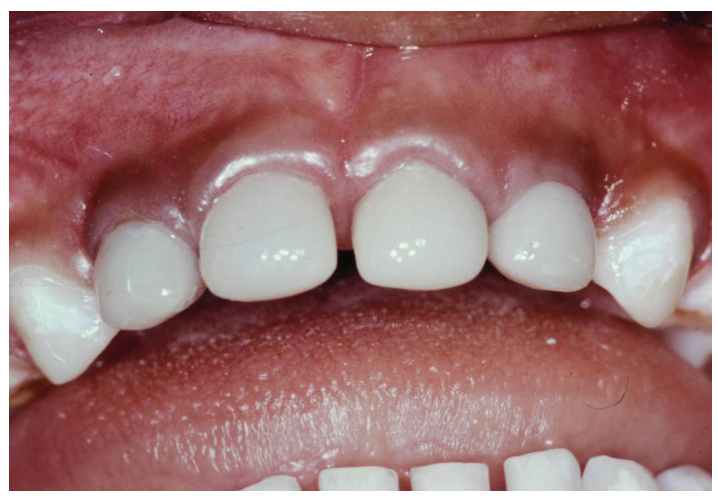

Figura 5: (A) Coroas de resina acrílica prensadas cimentadas. Vista frontal. 


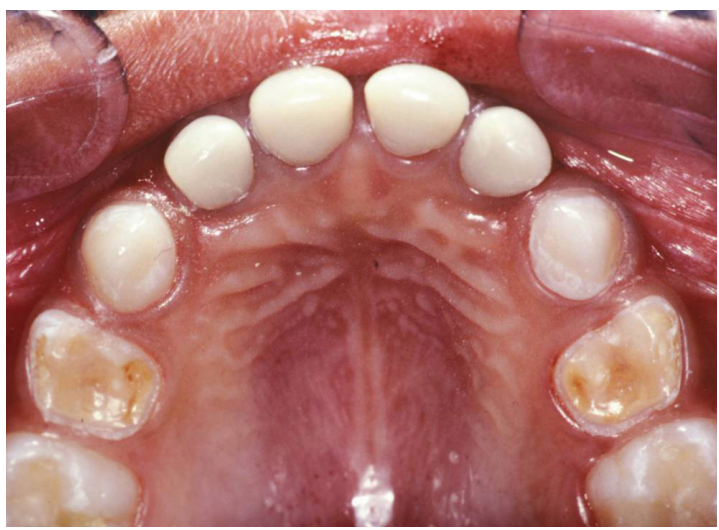

FIGURA 5 (B): Coroas de resina acrílica prensadas cimentadas. Vista oclusal.

O tratamento reabilitador da região anterior foi finalizado totalizando três sessões. Os responsáveis pela criança foram orientados quanto ao controle da dieta e higiene bucal. Também foram alertados para retornarem aos exames periódicos programados de acordo com o planejamento das manutenções preventivas.

\section{Discussão}

São várias as opções de reabilitação em dentição decídua que se apresentam comprometidas, devido principalmente à cárie aguda da infância. ${ }^{4}$ Por isso, é imprescindível que o profissional tenha conhecimento de suas indicações e vantagens.

Dentes decíduos com extensas destruições requerem um reforço intracanal. Isso ocorre, pois, muitas vezes a estrutura coronária remanescente não suporta forças mastigatórias e não possui retenção para a restauração do dente. É importante salientar que devem ser respeitados os princípios da terapia endodôntica e retenção intracanal até $1 / 3$ da raiz, além do acompanhamento periódico. ${ }^{4}$

O reforço intrarradicular é contraindicado para dentes anteriores de pacientes com bruxismo, mordida profunda e em pacientes com grande perda de dimensão vertical, por não apresentarem espaço protético suficiente para a reconstrução. A falta de cuidado diante desse detalhe pode levar à sobrecarga de forças sobre o elemento dentário em questão, podendo provocar a reabsorção patológica da raiz, acelerando sua esfoliação, ou ainda levando ao insucesso pela fratura ou queda da restauração e reforço intrarradicular. Antes de indicar a reconstrução dos dentes anteriores, deve ser avaliado se existe espaço para tal, deste modo se o paciente tiver perdido a dimensão vertical, esta deve ser recuperada antes da reabilitação anterior. ${ }^{5}$
O reforço intracanal realizado com pinos com fio ortodôntico foi bastante utilizado na clínica odontopediátrica. Porém, este método consiste na formação de tensões nas paredes internas, que podem levar à fratura do remanescente radicular. ${ }^{6}$

Os núcleos de preenchimento em resina ou cimento de ionômero de vidro possuem função satisfatória, entretanto os pinos metálicos são mais adequados. Isto porque estes são mais resistentes à fratura, uma vez que os outros materiais não têm resistência mecânica adequada. ${ }^{7} \mathrm{O}$ uso de pinos biológicos intracanais, ou seja, pinos obtidos por meio de fragmento dentário do próprio paciente ou de um banco de dentes, é uma nova opção. Sua maior desvantagem é a necessidade de um banco de dentes e a aceitação por parte da família. ${ }^{8}$

$\mathrm{Na}$ literatura são encontradas opções diversas de coroas para reabilitação de dentes decíduos anteriores. As principais citadas são as coroas de aço e policarbonato, as quais estão em desuso, ${ }^{9}$ coroas (matriz) de celuloide, facetas de resina, coroas biológicas, coroas prensadas. ${ }^{10,11}$ No caso clínico relatado, optou-se pela reabilitação com núcleos metálicos e coroas em resina acrílica prensadas. Esta técnica requer uma fase laboratorial, o que consequentemente aumenta o custo final do tratamento. ${ }^{12}$ Porém, em odontopediatria é importante que o comportamento do paciente seja avaliado. A utilização desta técnica permitiu a diminuição do tempo de atendimento da criança não colaboradora em cada sessão clínica. O resultado estético foi muito favorável, além da vantagem de boa adaptação cervical.

\section{Conclusão}

A coroa de resina acrílica prensada com núcleo metálico fundido é uma opção de tratamento com resultados extremamente satisfatórios. Apesar de aumentar o custo final e o número de consultas, torna a duração das mesmas mais curtas, o que é interessante em odontopediatria.

\section{REFERÊNCIAS BIBLIOGRÁFICAS}

1. Navit S, Katiyar A, Samadi F, Jaiswal JN. Rehabilitation of severely mutilated teeth under general anesthesia in an emotionally immature child. J Indian Soc Pedod Prev Dent. 2010 jan-fev;54(1):11-9.

2. Usha M, Deepak V, Venkat S, Gargi M. Treatment of severely mutilated incisors: Challenge to the pedodontist. J Indian Soc Pedod Prev Dent. 2007;25(5):34-6. 
3. Motisuki C, Santos-Pinto L, Giro EMA. Restoration of severely decayed primary incisors using indirect composite resin restoration technique. Int J Paediatr Dent. 2005 jul;15(4):282-6.

4. Arora C, Aras M, Chitre V. Evaluation and comparison of retention of different aesthetic posts. J Indian Prosthodont Soc. 2006;6(2):82-9.

5. Oliveira LB, Peixoto LFS, Cristina Giovanetti Del Conte Zardetto CGDC, Corrêa MSNP5, Wanderley MT. Reabilitação de dentes decíduos anteriores com o uso de pinos de fibra de vidro. J Health Sci Inst. 2010;28(1):89-93.

6. Ramires-Romito ACD, Wanderley MT, Oliveira MDM, Imparato JCP, Corrêa MSNP. Biologic restoration of primary anterior teeth. Quintessence Int. 2000;31(6):405-11.

7. Wanderley MT, Ferreira SL, Rodrigues CR, Rodrigues Filho LE. Primary anterior tooth restoration using posts with macroretentive elements. Quintessence Int. 1999 jun;30(6):432-6.

8. Grewal N, Seth R. Comparative in vivo evaluation of restoring severely mutilated primary anterior teeth with biological post and crown preparation and reinforced composite restoration. J Indian Soc Pedod Prev Dent. 2008;26(4):141-8.

9. Mendes FM, Benedetto MS, Zardetto CGC, Wanderley MT, Corrêa MSNP. Resin composite restoration in primary anterior teeth using short-post technique and strip crowns: A case report. Quintessence Int. 2004;35(9):689-92.

10. Khatri A; Nandlal B, Srilatha. Comparative evaluation of shear bond strength of conventional composite resin and nanocomposite resin to sandblasted primary anterior stainless steel crown. J Indian Soc Pedod Prev Dent. 2007apr-jun;25(2):82-5

11. Jain M, Singla S, Bhushan B, Kumar S, Bhushan A. Esthetic rehabilitation of anterior primary teeth using polyethylene fiber with two different approaches. J Indian Soc Pedod Prev Dent. 2011;29(4):327-32.

12. Galassi MAS, Borsatto MC, Santos-Pinto LMS. Reabilitação de dentes decíduos anteriores: relato e acompanhamento de um caso clínico. 1999;2(7):175-8

Submetido em: $11 / 9 / 2012$

Aceito em: 25/11/2012 